\title{
Drug pipeline 3Q21-mixed news for genetic therapies
}

Zydus Cadila Healthcare's COVID-19 vaccine-the world's first DNA vaccine approved for humans-got the nod in the form of an Emergency Use Authorization from India's Central Drugs Standard Control Organisation. Regeneron's monoclonal antibody (mAb) cocktail Ronapreve continued to shine in clinical trials, with the latest study reporting it protects people exposed to SARS-CoV-2. Meanwhile, the European Medicines Agency (EMA) got out ahead of the US Food and Drug Administration (FDA) with registrations of the first drug for achondroplasia (BioMarin's Voxzogo), the first bispecific mAb for psoriasis (UCB's Bimzelx), and the first gene therapy for cerebral adrenoleukodystrophy (Bluebird Bio's lentiviral product Skysona). All was not plain sailing for Bluebird, however, with the FDA putting a clinical hold on the program due to concerns over possible insertional mutagenesis leading to myelodysplastic syndrome. Preclinical liver tumorigenicity concerns added to previous hepatotoxicity, thrombotic microangiopathy and neurotoxicity issues associated with adeno-associated viral (AAV) vector gene therapies, as the FDA placed a clinical hold on BioMarin's AAV-5 gene therapy for phenylketonuria. A phase 1/2 trial of Arrowhead's cystic fibrosis siRNA drug Aro-ENAC was also halted due to a preclinical signal of lung inflammation.

\section{FDA approvals by drug type}

Approvals for the first three quarters almost equal the total for all of last year.

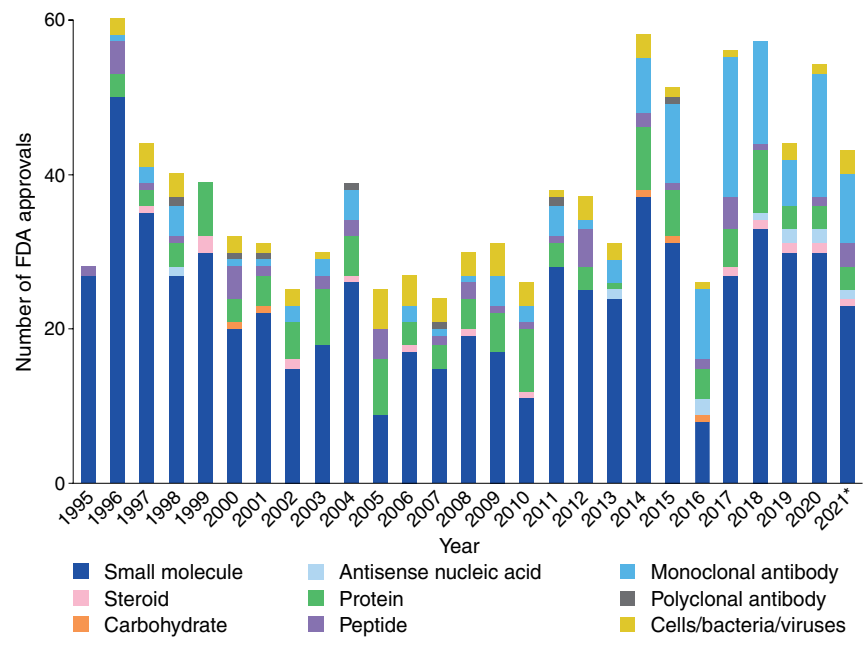

*Partial year to 30 June.

\section{Notable regulatory setbacks (3Q21)}

\begin{tabular}{|c|c|c|}
\hline Drug / company & Indication & Drug information \\
\hline $\begin{array}{l}\text { ARO-ENaC/ } \\
\text { Arrowhead } \\
\text { Pharmaceuticals }\end{array}$ & Cystic fibrosis & $\begin{array}{l}7 / 2 / 2021 \text { Company voluntarily halts phase } 2 \\
\text { clinical trial of this inhaled nebulized solution of } \\
\text { a small interfering (si)RNA targeting SCNN1A } \\
\text { mRNA, encoding the epithelial sodium channel } \\
\alpha \text {-subunit (aENaC), encapsulated in peptide E } \\
\text { (K16GACSERSMNFCG)-targeted DOTMA:DOPE } \\
\text { liposomes, after a toxicology study in rats showed } \\
\text { local lung inflammation }\end{array}$ \\
\hline $\begin{array}{l}\text { Teplizumab / } \\
\text { Provention Bio }\end{array}$ & $\begin{array}{l}\text { Diabetes mellitus, } \\
\text { type I }\end{array}$ & $\begin{array}{l}7 / 2 / 2021 \mathrm{FDA} \text { issues a complete response letter } \\
\text { for this humanized IgG1 mAb with an Fc engineered } \\
\text { with leucine-to-alanine substitutions at residues } \\
234 \text { and } 235 \text { (in the } \mathrm{CH} 2 \text { region) to abolish Fc } \\
\text { receptor binding directed against the } \mathrm{CD} 3 \varepsilon \text {-chain } \\
\text { expressed on mature T lymphocytes }\end{array}$ \\
\hline
\end{tabular}

\begin{tabular}{|c|c|c|}
\hline Drug / company & Indication & Drug information \\
\hline $\begin{array}{l}\text { Lenti-D } \\
\text { (elivaldogene } \\
\text { autotemcel)/ } \\
\text { Bluebird Bio }\end{array}$ & Adrenoleukodystrophy & $\begin{array}{l}8 / 9 / 2021 \text { FDA puts a clinical hold on this } \\
\text { autologous hematopoietic CD } 34^{+} \text {stem cells } \\
\text { transduced with a lentiviral vector encoding human } \\
A B C D 1 \text { CDNA due to myelodysplastic syndrome } \\
\text { potentially mediated by vector insertion }\end{array}$ \\
\hline $\begin{array}{l}\text { Verdiperstat / } \\
\text { Biohaven } \\
\text { Pharmaceuticals }\end{array}$ & $\begin{array}{l}\text { Multiple system } \\
\text { atrophy }\end{array}$ & $\begin{array}{l}\text { 9/27/2021 Company announces suspension } \\
\text { of its phase } 3 \text { trial of this oral, small-molecule, } \\
\text { brain-penetrant, irreversible inhibitor of } \\
\text { myeloperoxidase due to lack of statistically } \\
\text { significant improvement }\end{array}$ \\
\hline $\begin{array}{l}\text { BMN } 307 / \\
\text { BioMarin }\end{array}$ & Phenylketonuria & $\begin{array}{l}\text { 9/6/2021 FDA puts a clinical hold on this an AAV- } \\
5 \text {-vectored gene therapy containing the gene for } \\
\text { phenylalanine hydroxylase after mice developed } \\
\text { liver tumors in a preclinical study }\end{array}$ \\
\hline $\begin{array}{l}\text { Ad26.Mos4.HIV } \\
\text { vaccine / Johnson \& } \\
\text { Johnson }\end{array}$ & HIV & $\begin{array}{l}8 / 31 / 2021 \text { Company announced that this } \\
\text { tetravalent recombinant replication-deficient } \\
\text { adenovirus } 26 \text { (Ad26) vectored vaccine comprising } \\
\text { two Ad26 vectors containing a mosaic insert of } \\
\text { the HIV envelope (Env) sequence and two Ad26 } \\
\text { vectors containing mosaic inserts of Gag and } \\
\text { Pol sequences, along with a booster dose of the } \\
\text { trimeric clade C glycoprotein } 140 \text {, achieved only } \\
25.2 \% \text { efficacy in a phase } 2 \text { trial }\end{array}$ \\
\hline $\begin{array}{l}\text { Ultomiris } \\
\text { (ravulizumab- } \\
\text { cwvz)/ } \\
\text { AstraZeneca }\end{array}$ & $\begin{array}{l}\text { Amyotrophic lateral } \\
\text { sclerosis }\end{array}$ & $\begin{array}{l}8 / 20 / 2021 \text { Company discontinues phase } 3 \text { trial of } \\
\text { this long-acting humanized lgG } 2 / \mathrm{lgG} 4 \text { anti-C } 5 \mathrm{mAb} \\
\text { with an Fc engineered for increased human FcRn } \\
\text { affinity (Xtend technology) due to lack of efficacy }\end{array}$ \\
\hline $\begin{array}{l}\text { Vicineum } \\
\text { (oportuzumab } \\
\text { monatox-qqrs)/ } \\
\text { Sesen Bio }\end{array}$ & Bladder cancer & $\begin{array}{l}8 / 16 / 2021 \mathrm{FDA} \text { issues a complete response } \\
\text { letter for this fusion of anti-EpCAM humanized } \\
\text { single-chain variable fragment (scFv) linked to } \\
\text { a truncated form of Pseudomonas exotoxin A, } \\
\text { requesting additional clinical and statistical data } \\
\text { and analyses and citing chemistry, manufacturing } \\
\text { and control issues }\end{array}$ \\
\hline $\begin{array}{l}\text { Aduhelm } \\
\text { (aducanumab) / } \\
\text { Biogen }\end{array}$ & Alzheimer's disease & $\begin{array}{l}8 / 11 / 2022 \text { The US Department of Veterans Affairs } \\
\text { announces it will not include in its formulary this } \\
\text { fully human IgG1 mAb against a conformational } \\
\text { epitope on } \beta \text {-amyloid plaques }\end{array}$ \\
\hline $\begin{array}{l}\text { COVID vaccine / } \\
\text { Sanofi }\end{array}$ & COVID-19 prevention & $\begin{array}{l}9 / 29 / 2021 \text { Company halts testing of } \\
\text { this mRNA-based vaccine comprising } \\
\text { the stabilized pre-fusion form of the } \\
\text { SARS-CoV-2 spike glycoprotein delivered by } \\
\text { a lipid nanoparticle comprising ionizable lipid, } \\
\text { phosphatidylethanolamine, cholesterol and } \\
\text { polyethylene glycol-lipid }\end{array}$ \\
\hline
\end{tabular}

DOTMA, 1,2-di-O-octadecenyl-3-trimethylammonium propane; DOPE, 1,2-dioleoyl-sn-glycero3-phosphoethanolamine; IgG, immunoglobulin G; Fc, immunoglobulin constant fragment; FcRn, neonatal Fc receptor; C5, complement protein 5; EpCAM, epithelial cell adhesion molecule. Source: BioMedTracker, a service of Sagient Research (http://www.biomedtracker.com) and company press releases 


\section{Notable clinical trial results (3Q21)}

\begin{tabular}{|c|c|c|}
\hline Drug / company & Indication & Drug information \\
\hline $\begin{array}{l}\text { Tebentafusp / } \\
\text { Immunocore }\end{array}$ & Uveal melanoma & $\begin{array}{l}9 / 22 / 2021 \text { In a phase } 3 \text { open-label trial, patients } \\
\text { receiving this soluble bispecific fusion of a high-affinity } \\
\text { monoclonal T-cell receptor (mTCR) specific for gp100 } \\
\text { (melanocytic protein) in the context of HLA-A*0201 and } \\
\text { an anti-CD3 scFv fragment had } 73 \% \text { overall survival at } \\
\text { one year compared with } 59 \% \text { in control group (N. Engl. J. } \\
\text { Med } 385,1196-1206,2021 \text { ) }\end{array}$ \\
\hline $\begin{array}{l}\text { Uproleselan / } \\
\text { Glycomimetics }\end{array}$ & $\begin{array}{l}\text { Acute myelogenous } \\
\text { leukemia }\end{array}$ & $\begin{array}{l}9 / 20 / 2021 \text { In a phase } 2 / 3 \text { study of this synthetic } \\
\text { glycomimetic small-molecule E-selectin antagonist, } \\
\text { which sensitizes leukemic cells to the chemotherapeutic } \\
\text { cytarabine by mobilizing them from their protective bone } \\
\text { marrow niche, patients showed increased remission rates } \\
\text { and improved survival (Blood https://doi.org/10.1182/ } \\
\text { blood.2021010721, 2021) }\end{array}$ \\
\hline $\begin{array}{l}\text { Ensartinib / } \\
\text { Xcovery }\end{array}$ & $\begin{array}{l}\text { Non-small cell lung } \\
\text { cancer }\end{array}$ & $\begin{array}{l}9 / 2 / 2021 \text { In a phase } 3 \text { open label randomized trial of this } \\
\text { aminopyridazine-based small-molecule ALK inhibitor, } \\
\text { the drug doubled progression-free survival compared } \\
\text { with that seen with the small-molecule kinase inhibitor } \\
\text { crizotinib (JAMA Oncol. https://doi.org/10.1001/ } \\
\text { jamaoncol.2021.3523, 2021) }\end{array}$ \\
\hline $\begin{array}{l}\text { Lumevoq / } \\
\text { GenSight }\end{array}$ & $\begin{array}{l}\text { Leber's hereditary } \\
\text { optic neuropathy }\end{array}$ & $\begin{array}{l}8 / 31 / 2021 \text { In a long-term follow up study of this } \\
\text { AAV-2 gene therapy encoding the wild-type NADH } \\
\text { dehydrogenase } 4 \text { gene (ND4), treated patients showed } \\
\text { progressive and sustained improvement of best-corrected } \\
\text { visual acuity up to } 52 \text { months (J. Neuroophthalmol. } 41 \text {, } \\
\text { 309-315, 2021) }\end{array}$ \\
\hline $\begin{array}{l}\text { Balstilimab / } \\
\text { Agenus }\end{array}$ & Cervical cancer & $\begin{array}{l}8 / 25 / 2021 \text { In a phase } 2 \text { study of this fully human IgG4 } \\
\text { mAb targeting programmed death } 1 \text { receptor (PD- } 1 \text { ), } \\
\text { treated patients had a } 20 \% \text { response rate with median } \\
\text { duration not reached at } 14.6 \text { months (Gynecologic Oncol. } \\
\text { https://doi.org/10.1016/j.ygyno.2021.08.018, 2021) }\end{array}$ \\
\hline $\begin{array}{l}\text { Apabetalone / } \\
\text { Resverlogix }\end{array}$ & $\begin{array}{l}\text { Cardiovascular } \\
\text { disease }\end{array}$ & $\begin{array}{l}8 / 24 / 2021 \text { In a randomized trial with patients at risk } \\
\text { for a cardiovascular event, patients with low baseline } \\
\text { scores receiving this small-molecule bromodomain } \\
\text { and extraterminal domain (BET) inhibitor that reduces } \\
\text { vascular inflammation and calcification scored better } \\
\text { on cognitive tests (J. Alzheimers Dis. https://doi. } \\
\text { org/10.3233/JAD-210570, 2021) }\end{array}$ \\
\hline $\begin{array}{l}\text { Miplyffa } \\
\text { (arimoclomol) / } \\
\text { CytRx }\end{array}$ & $\begin{array}{l}\text { Niemann-Pick } \\
\text { disease }\end{array}$ & $\begin{array}{l}8 / 21 / 2021 \text { In a randomized, placebo-controlled trial, } \\
\text { patients receiving this small-molecule co-inducer of } \\
\text { heat shock protein HSF1 had a } 65 \% \text { relative reduction } \\
\text { in annual progression }(J . \text { Inherit. Metab. Dis. https://doi. } \\
\text { org/10.1002/jimd.12428, 2021) }\end{array}$ \\
\hline $\begin{array}{l}\text { RVT-802/ } \\
\text { Enzyvant } \\
\text { Therapeutics }\end{array}$ & DiGeorge syndrome & $\begin{array}{l}8 / 4 / 2021 \text { In ten single-arm, open-label studies in which } \\
105 \text { patients were treated with allogeneic cultured } \\
\text { decapsulated thymic tissue, } 77 \% \text { survived one year } \\
\text { (J. Allergy Clin. Immunol. https://doi.org/10.1016/j. } \\
\text { jaci.2021.06.028, 2021) }\end{array}$ \\
\hline $\begin{array}{l}\text { Rinvoq } \\
\text { (upadacitinib) / } \\
\text { AbbVie }\end{array}$ & Atopic dermatitis & $\begin{array}{l}8 / 4 / 2020 \text { In a phase } 3 \text { randomized comparator } \\
\text { controlled study, this small-molecule inhibitor } \\
\text { of Janus kinase } 1 \text { (JAK1) showed superiority to } \\
\text { dupilumab (JAMA Dermatol. https://doi.org/10.1001/ } \\
\text { jamadermatol.2021.3023, 2021) }\end{array}$ \\
\hline $\begin{array}{l}\text { Masitinib / AB } \\
\text { Science }\end{array}$ & $\begin{array}{l}\text { Amyotrophic lateral } \\
\text { sclerosis }\end{array}$ & $\begin{array}{l}7 / 19 / 2021 \text { In a randomized, placebo-controlled trial, this } \\
\text { oral, small-molecule inhibitor of wild-type and mutated } \\
\text { forms of c-Kit (stem cell factor receptor), Lyn and } \\
\text { platelet-derived growth factor receptor kinases prolonged } \\
\text { survival by over two years provided treatment was begun } \\
\text { before severe impairment (Ther. Adv. Neurol. Dis. https:// } \\
\text { doi.org/10.1177/17562864211030365, 2021) }\end{array}$ \\
\hline $\begin{array}{l}\text { Kineret } \\
\text { (anakinra)/ } \\
\text { Swedish Orphan } \\
\text { Biovitrum }\end{array}$ & COVID-19 treatment & $\begin{array}{l}9 / 3 / 2021 \text { In phase } 3 \text { randomized, placebo-controlled trial } \\
\text { of patients at risk for severe disease, this recombinant, } \\
\text { non-glycosylated form of human interleukin-1 receptor } \\
\text { antagonist reduced the odds of worse outcome to } 0.36 \text { at } \\
\text { day } 28 \text { (Nat. Med. https://doi.org/10.1038/s41591-021- } \\
01499-z, 2021)\end{array}$ \\
\hline $\begin{array}{l}\text { Ronapreve } \\
\text { (casirivimab } \\
\text { imdevimab)/ } \\
\text { Regeneron }\end{array}$ & COVID-19 prevention & $\begin{array}{l}8 / 4 / 2021 \text { In a phase } 3 \text { trial of people exposed in the home } \\
\text { to COVID-19, this cocktail of two neutralizing human IgG1 } \\
\text { mAbs targeting SARS-CoV- } 2 \text { spike glycoprotein epitopes } \\
\text { reduced the incidence of systemic infection by } 77 \% \text { (N. } \\
\text { Engl. J. Med. https://doi.org/10.1056/NEJMoa2109682, } \\
\text { 2021) }\end{array}$ \\
\hline
\end{tabular}

ALK, anaplastic lymphoma kinase. Source: BioMedTracker, a service of Sagient Research

(http://www.biomedtracker.com)

\section{Notable drug approvals (3Q21)}

\begin{tabular}{|c|c|c|}
\hline Drug/company & Indication & Drug information \\
\hline $\begin{array}{l}\text { Opzelura (ruxolitinib) / } \\
\text { Incyte }\end{array}$ & Atopic dermatitis & $\begin{array}{l}9 / 21 / 2921 \text { FDA approves } \\
\text { this small-molecule JAK/ } \\
\text { STAT inhibitor formulated as } \\
\text { a cream }\end{array}$ \\
\hline $\begin{array}{l}\text { Tivdak (tisotumab } \\
\text { vedotin-tftv) / Seagen }\end{array}$ & Cervical cancer & $\begin{array}{l}9 / 20 / 2021 \text { FDA grants } \\
\text { accelerated approval to this } \\
\text { human tissue factor } \lg \mathrm{G} 1 \mathrm{\kappa} \mathrm{mAb} \\
\text { targeting tissue factor conjugated } \\
\text { to MMAE via a protease- } \\
\text { cleavable linker }\end{array}$ \\
\hline
\end{tabular}

Exkivity (mobocertinib) / Non-small-cell lung cancer Takeda

\section{9/15/2021 FDA grants}

accelerated approval to this

small-molecule selective

tyrosine kinase inhibitor targeting EGFR and human

EGFR2 exon insertion 2

Voxzogo (vosoritide)/ Achondroplasia

BioMarin

8/27/2021 EMA approves this

stabilized 39-residue analog

of C-type natriuretic peptide

containing 17 extra amino acids

(PGQEHPNARKYKGANKK)

appended to the native hormone's

$\mathrm{N}$ terminus

Bimzelx (bimekizumab) / Psoriasis

UCB

8/24/2021 EMA approved

this humanized bispecific $\lg \mathrm{G} 1$

$\mathrm{mAb}$ that neutralizes both IL-17A and IL-17F

Korsuva (difelikefalin) / Pruritus

Cara Therapeutics

Evrenzo (roxadustat) / Anemia due to chronic renal

AstraZeneca

failure

Nexviazyme

(avalglucosidase

Pompe diseas

alfa-ngpt) / Sanofi

Saphnelo (anifrolumab-

Systemic lupus erythematosus

fnia) / AstraZeneca

Skysona (Lenti-D,

(elivaldogene

autotemcel) /

Bluebird Bio

Cerebral adrenoleukodystrophy

8/23/2021 FDA approves this

small-molecule $\kappa$-opioid receptor agonist

8/19/2021 EMA approves

this second-generation

hypoxia-inducible factor prolyl

hydroxylase inhibitor

8/6/2021 FDA approves

this $\alpha$-glucosidase enzyme

replacement therapy

targeting mannose-6-phophate

receptor to facilitate cellular

uptake

$7 / 30 / 2021$ FDA approves this fully human IgG1 mAb targeting interferon- $\alpha$ receptor 1

7/21/2021 EMA approves these autologous hematopoietic $\mathrm{CD} 34^{+}$ stem cells transduced with a lentiviral vector encoding human $A B C D 1$ CDNA under the control of a modified enhancer/promoter of myeloproliferative sarcoma virus

Bylvay (odevixibat) / Progressive familial intrahepatic Albireo Pharma cholestasis

7/20/2021, 7/19/2021 FDA and EMA approve this smallmolecule inhibitor of ileal bile acid transporter

Rezurock (belumosudil) / Graft versus host disease Kadmon Holdings treatment

7/16/2021 FDA approves under its real-time approval review process this small-molecule selective oral inhibitor of Rho-associated coiled-coil kinase 2 (ROCK2) involved in inflammatory responses

Zy-CoV-D / Zydus Cadila COVID-19 prevention 8/20/2021 India's Drugs Controller General grants an EUA to this DNA vaccine encoding the spike glycoprotein of SARS-CoV-2 and IgE signal peptide delivered intradermally via the PharmaJet Tropis ID fluid-stream injection device

ABCD gene, ATP-binding cassette D; EGFR, epidermal growth factor receptor; EUA, Emergency Use Authorization; IgE, immunoglobulin E; IL, interleukin; MMAE, monomethyl auristatin E. Source: BioMedTracker, a service of Sagient Research (http://www.biomedtracker.com) 


\section{Upcoming catalysts (1Q22)}

\begin{tabular}{|c|c|c|}
\hline Drug / company & Indication & Drug information \\
\hline Tezepelumab / Amgen & Asthma & $\begin{array}{l}\text { 1/10/2022 FDA PDUFA date for this } \\
\text { human IgG2 } \mathrm{\kappa} \text { mb against thymic } \\
\text { stromal lymphopoietin }\end{array}$ \\
\hline Maribavir / Takeda & Cytomegalovirus infection & $\begin{array}{l}1 / 21 / 2021 \text { FDA PDUFA date for this } \\
\text { selective ATP competitor of viral DNA } \\
\text { polymerase }\end{array}$ \\
\hline $\begin{array}{l}\text { Oteseconazole / } \\
\text { Mycovia } \\
\text { Pharmaceuticals }\end{array}$ & Non-systemic fungal infections & $\begin{array}{l}\text { 1/27/2022 FDA PDUFA date for this } \\
\text { small-molecule inhibitor of lanosterol } \\
\text { demethylase (CYP51), an enzyme } \\
\text { involved in the synthesis of fungal cell } \\
\text { wall sterols }\end{array}$ \\
\hline $\begin{array}{l}\text { Mavacamten / Bristol } \\
\text { Myers Squibb }\end{array}$ & Hypertrophic cardiomyopathy & $\begin{array}{l}1 / 28 / 2022 \text { FDA PDUFA date for this } \\
\text { first-in-class small-molecule allosteric } \\
\text { modulator of cardiac myosin }\end{array}$ \\
\hline Faricimab / Roche & $\begin{array}{l}\text { Diabetic macular edema/ } \\
\text { wet age-related macular } \\
\text { degeneration }\end{array}$ & $\begin{array}{l}\text { 1/31/2022 FDA PDUFA date for this } \\
\text { IgG1 bispecific } m A b \text { against vascular } \\
\text { endothelial growth factor } A \text { and } \\
\text { angiopoietin-2 }\end{array}$ \\
\hline Mitapivat / Agios & Pyruvate kinase deficiency & $\begin{array}{l}\text { 2/17/2022 FDA PDUFA date for this } \\
\text { first-in-class small-molecule allosteric } \\
\text { activator of wild-type and mutated } \\
\text { pyruvate kinase }\end{array}$ \\
\hline $\begin{array}{l}\text { Bardoxolone methyl/ } \\
\text { Reata Pharmaceuticals }\end{array}$ & Alport syndrome & $\begin{array}{l}\text { 2/25/2022 FDA PDUFA date for } \\
\text { this small-molecule (synthetic } \\
\text { triterpenoid) activator of nuclear } \\
\text { factor erythroid 2-related factor } 2 \\
\text { (Nrf2), which suppresses NF-kB- and } \\
\text { STAT3-mediated inflammation }\end{array}$ \\
\hline $\begin{array}{l}\text { Roctavian (valoctocogene } \\
\text { roxaparvovec)/BioMarin }\end{array}$ & Hemophilia A & $\begin{array}{l}12 / 31 / 2021 \text { EMA's CHMP opinion due } \\
\text { date for this AAV- } 8 \text { vector encoding a } \\
\text { factor VIII gene therapy }\end{array}$ \\
\hline
\end{tabular}

PDUFA, Prescription Drug User Fee Act; CHMP, Committee for Medicinal Products for Human Use (Europe); IgG, immunoglobulin G. Source: BioMedTracker, a service of Sagient Research (http://www. biomedtracker.com)

\section{Laura DeFrancesco}

Senior Editor, Nature Biotechnology.

Published online: 9 November 2021

https://doi.org/10.1038/s41587-021-01119-8 